\title{
Blindstellen: ungleiche Entwicklung und ungleiche Mobilisierung in der EU
}

Alex Demirović und Thomas Sablowski haben in PROKLA 166 einen wichtigen Beitrag zur Krise in Europa geschrieben und darin grundlegende politische Schlussfolgerungen formuliert. Hierbei heben sie als Ziel besonders hervor, „die Einheit der subalternen Klassen in Europa durch gemeinsame Kämpfe herzustellen." (Demirović/Sablowski 2012: 103). Meines Erachtens überschätzen die beiden Autoren die Möglichkeiten einer gemeinsamen Mobilisierung, da sie das Ausmaß der ungleichen Entwicklung in der EU unterschätzen. Die zentrale Bedeutung des monetären Regimes, speziell der Währungsunion, bei der Zementierung der ungleichen Entwicklungsmuster einerseits und der gesellschaftlichen Verhältnisse andererseits wird nur unzureichend thematisiert. Diese Blindstellen teilen sie mit vielen anderen linken SozialwissenschaftlerInnen in Westeuropa.

Die Akkumulationsregime in der EU können für die letzten zwanzig bis dreißig Jahre nicht allein als finanzgetrieben gekennzeichnet werden. Vielmehr hat sich aufbauend aufälteren Mustern ungleicher europäischer Entwicklung eine Verschränkung von exportorientierter industrieller Entwicklung und Kapitalexport auf der einen Seite und ausgeprägter Finanziarisierung und Waren- und Kapitalimport auf der anderen Seite herausgebildet (Becker/ Jäger 2009). Hierbei ist der exportorientierte Block um die deutsche Ökonomie herum gebildet und umfasst vor allem die Benelux-Länder, Österreich, Norditalien sowie seit den 1990er Jahren auch die Tschechische Republik, die Slowakei, Slowenien, Polen sowie Ungarn. Ähnliche Muster sind auch in den skandinavischen Ländern zu erkennen. Den Gegenpol stellen hochgradig finanziarisierte und importabhängige Ökonomien in Südeuropa sowie, seit den 1990er Jahren, auch in Südosteuropa und den Baltischen Ländern dar. Das Charakteristikum beträchtlicher Importabhängigkeit weist ebenfalls die britische Ökonomie auf, in ihrem Zentrum steht allerdings - im Gegensatz zu den peripheren Ökonomien im Süden und Osten Europas - ein eigenes Finanzzentrum.

\section{Ungleiche Entwicklung}

Die ungleichen Entwicklungsmuster in Europa gehen historisch weit zurück (vgl. z.B. Weissenbacher 2007: 42 ff.). Der europäische Integrationsprozess brach die Ungleichheit der Entwicklungsmuster nicht auf, sondern akzentuierte sie weiter. Der EU-Beitritt war für die südeuropäischen Ökonomien bereits in den 1980er Jahren mit einer teilweisen Deindustrialisierung verbunden (vgl. z.B. López/Rodríguez 2010: 161 ff., Santos/ Jacinto 2006, Stathakis 2010: 110). López und Rodríguez (2010: $161 \mathrm{f}$.) heben hervor, dass die industriepolitischen Spielräume Spaniens durch den EU-Beitritt empfindlich 
beschnitten wurden und industriepolitische Programme nicht Bestandteil der EURegionalpolitik wurden. Dieser Zuschnitt der Wirtschaftspolitik lag im Interesse der deutschen Exportindustrie. Damit erfolgte eine zunehmende Orientierung der Kapitalakkumulation in Spanien bereits damals in Richtung auf Immobilienwirtschaft und Tourismus. Diese Weichenstellung wurde durch die damalige Überbewertung der Peseta, die durch Verbilligung der Importe die binnenorientierte Industrie schwächte, noch befördert. Auch die griechische Bourgeosie verlegte sich, vor allem ab den 1990er Jahren, vermehrt auf das Import-, Finanz- und Dienstleistungsgeschäft. Kennzeichnend ist für diese Entwicklung beispielsweise die Sfakianakis-Gruppe, die sich in den 1960er Jahren noch in der Busfertigung engagierte, angesichts rückläufiger Profite in den 1980er Jahren jedoch verstärkt ins Importgeschäft einstieg und zum führenden Autoimporteur Griechenlands aufstieg (Fouskas 2012:34).

Der Beitritt zur Eurozone verstärkte diese Muster der ungleichen Entwicklung. Den Finanzinteressen ging es bei der Schaffung des Euros um eine relativ starke internationale Reservewährung. Darüber hinaus gab es aber auch noch unterschiedliche Interessenkonstellationen in den europäischen Zentrums- und Peripherieländern. Das deutsche Industriekapital sah in der Schaffung des Euro die Möglichkeit, der Stärkung der Konkurrenzfähigkeit in anderen Zentrumsökonomien (vor allem Frankreich) und in Ökonomien der südeuropäischen Peripherie mittels Währungsabwertung einen Riegel vorzuschieben. Es ging dem deutschen Industriekapital also vor allem um eine Stärkung der eigenen Exportposition - und dieses Ziel wurde auch erreicht (vgl. Bellofiore et al. 2010). Spiegelbildlich verloren die südeuropäischen Länder den letzten verbliebenen Schutzmechanismus für die einheimische verarbeitende Industrie.
Spanien, Portugal und Griechenland gehörten zu jenen EU-Ländern, in denen der Anteil der Industrie seit Ende 1990er Jahre am nationalen BIP besonders stark gefallen ist. In Griechenland war 2008 nach dem Finanzzentrum Luxemburg sowie Zypern der Industrieanteil am BIP von 13,3\% am drittniedrigsten unter den EU-Ländern (Becker/Jäger 2010: 27, Tab. 1).

Die strukturellen Divergenzen wurden durch die deutsche Politik der Lohndeflation - in erster Linie über die Hartz IVGegenreformen und die Förderung der Herausbildung eines Niedriglohnsektors vor allem durch die rot-grüne Bundesregierung - noch gefördert. Dafür bekamen die südeuropäischen Länder mit dem Euro Zugang zu billigeren Krediten. In den südeuropäischen Ländern sank das Zinsniveau signifikant. Hierdurch erhielt die finanziarisierte Akkumulation, vor allem in Spanien und Griechenland, neuen Auftrieb. Spanien entwickelte sich zum Musterfall eines Akkumulationsmodells, das durch einen kreditfinanzierten Immobilien- und Bauboom getragen war (López/Rodríguez 2010). Auch in Portugal spielte die hohe Privatverschuldung für die Akkumulation eine wichtige Rolle, ohne allerdings - angesichts eines bereits beträchtlichen Ausgangsniveaus - eine so stark treibende Kraft wie in Spanien entfalten zu können (vgl. Rodrigues/Reis 2012). In Griechenland stieg die Privatverschuldung ebenfalls, doch liefen hier kreditfinanzierte Impulse stärker über das staatliche Budget (vgl. z.B. Stathakis 2010, Tsarouhas 2012). Insofern könnte der griechische Fall als eine stärker etatistische Form der abhängigen Finanziarisierung gekennzeichnet werden.

Die ungleichen Entwicklungsprozesse drückten sich in einer ungleichen Entwicklung der Leistungsbilanzen aus. Spiegelbildlich zum starken Anstieg der deutschen Leistungsbilanzüberschüsse ab dem Jahr 
2000 stiegen die Leistungsbilanzdefizite der südeuropäischen Länder, besonders stark im Fall Griechenland, das im Jahr 2007 ein Leistungsbilanzdefizit von fast $15 \%$ des BIP - also in etwa des Dreifachen der als kritisch geltenden Grenze - erreichte (Kaltenbrunner 2012: 116). Die griechischen Exporte machten 2008 nur mehr 28,6\% der Importe aus, gegenüber ohnehin schon mageren 35,1\% im Jahr 2000 (Fouskas 2012: 33, Tab. 1). Die entstehende Lücke musste durch Schuldaufnahme im Ausland gefüllt werden. Entsprechend exorbitant fällt das Verhältnis Auslandsverschuldung/BIP derzeit aus: Griechenland 179,6\%, Spanien $165,3 \%$ und Portugal 231,5\% (Daten für 2010; Hrvatska narodna banka 2011: 13, Tab.). Der Finanziarisierungs- und Akkumulationsprozess war völlig vom Zufluss von Auslandskapital abhängig. Finanzgruppen aus den Zentrumsökonomien, vor allem Frankreich und Deutschland, partizipierten an der abhängigen Finanziarisierung in Südeuropa und stimulierten diese. Die Finanzströme finanzierten auch die Exporte der Leistungsbilanzüberschussländer, vor allem Deutschlands. Mit ihrem Austrocknen drohten absehbar ein Kollaps der Akkumulationsmodelle sowie eine offene Zahlungsbilanzkrise.

Analoge Tendenzen waren in den baltischen und südosteuropäischen Ländern zu beobachten. Auch hier zementierte die monetäre Norm Formen abhängiger, finanziarisierter Entwicklung. Diese Länder waren zwar nicht direkt Mitglied der Eurozone, aber durch das Wechselkurs-Regime und verbreitete Formen der informellen Euroisierung eng an die Eurozone angebunden. Bulgarien, Estland und Litauen optierten für sogenannte Currency Boards, bei denen einen fixer Wechselkurs zur Leitwährung fixiert ist, die Geldmenge an den Devisenbestand gekoppelt ist und keine Spielräume für eine nationalstaatliche Geldpolitik mehr bestehen. Lettland führte ein extrem rigides Wechselkursregime ein, das sich in den realen Wirkungen kaum von einem Currency Board unterschied. Rumänien behielt zwar einen flexiblen Wechselkurs bei, die Hochzinspolitik führte jedoch zu einer starken Währungsaufwertung (Becker 2007: 263 ff.). Diese Währungsarrangements begünstigten in Verbindung mit einem attraktiven Zinsniveau hohe Kapitalzuflüsse aus anderen EU-Ländern, vor allem kleineren Zentrumsökonomien wie Österreich, Schweden, Belgien (aber auch Griechenland spielte in Südosteuropa eine Rolle). Diese Flüsse finanzierten kaum Investitionen, sondern primär einen Immobilienboom, speziell in den Baltischen Ländern, aber auch privaten Konsum. Die Verschuldung der privaten Haushalte explodierte förmlich, die Kennzahl private Haushaltsverschuldung/BIP verdoppelte sich in der Regel zwischen 2003 und 2006 (Frangakis 2009: 64, Tab. 3.8). Die Banken - in den baltischen Ländern überwiegend in schwedischem Eigentum, in Rumänien mit sehr stark österreichischer Präsenz forcierten bei der inländischen Kreditvergabe Fremdwährungskredite. Hierdurch wurde die verschuldete Mittelschicht eng an das Wechselkursregime gebunden. Eine Währungsabwertung bedeutete für die DevisenkreditschulderInnen eine Aufwertung der Schulden in nationaler Währung und damit die Gefahr der Überschuldung (Becker 2010). Ähnlich wie in Südeuropa wurden die industriellen Strukturen in den baltischen und den südosteuropäischen Ländern geschwächt, am extremsten in Lettland. Die überbewerteten Wechselkurse führten zu einem Importboom und einer dramatischen Verschlechterung der Leistungsbilanzen. In Lettland und Bulgarien überstieg das Leistungsbilanzdefizit vor der Krise sogar 20\% des BIP (Becker 2010: 524). Die Auslandsverschuldung stieg stark 
an. Damit waren die Akkumulationsmodelle extrem abhängig vom Zufluss von Auslandskapital und krisenanfällig.

\section{Ungleiche Krisendynamik}

Die Wirkungen der Wirtschaftskrise waren in der EU ähnlich ungleich wie die Entwicklungsmuster. In den stark exportorientierten Ländern mit begrenzter innerer Finanziarisierung war die Kontraktion Ende 2008/Anfang 2009 vor allem durch den Exportkanal hervorgerufen und relativ stark. Danach setzte jedoch eine fragile (und vorübergehende) Erholung ein, nicht nur in der deutschen Ökonomie, sondern auch in Österreich und einigen der exportorientierten Ökonomien in Osteuropa. Je höher der Grad an innerer bzw. äußerer Finanziarisierung in den um den deutschen Kern gruppierten Ökonomien war, umso anhaltender waren die wirtschaftlichen Probleme.

Unmittelbar am stärksten war der wirtschaftliche Einbruch in den abhängig finanziarisierten Ökonomien Osteuropas. Hier blieben Kapitalzuflüsse aus oder kehrten sich angesichts des plötzlichen Liquiditätsbedarfs in den Zentrumsökonomien um. Damit war den finanziarisierten Modellen die Grundlage entzogen. Eine scharf prozyklische Politik verschärfte die Rezession. Die stärksten BIP-Rückgänge in der EU verzeichneten 2009 die baltischen Länder, Lettland allein in diesem Jahr 18\% (Becker 2010). In diesen Ländern explodierte die offene Arbeitslosigkeit. In Rumänien und Bulgarien dürfte sich ein Teil der Arbeitslosen in Kleinstlandwirtschaft geflüchtet haben, in Rumänien stieg die Arbeitsmigration noch mal stark an.

In den südeuropäischen Ländern der Eurozone erlebte das finanziarisierte Modell zwar auch einen Einbruch, mit der vollen Wucht von Problemen der internationalen (Re-)Finanzierung wurden sie allerdings erst ab etwa Anfang 2010 konfrontiert. Für eine Übergangszeit bildete die Mitgliedschaft in der Eurozone - und der damit verbundene Status als Anlageregion - einen gewissen Schutz vor externen Finanzierungsproblemen. Die Grundproblematik schwacher industrieller Strukturen, hoher Leistungsbilanzdefizite und eines permanenten externen Finanzierungsbedarfs war jedoch ähnlich. Das galt auch für die Krisendynamik. Die offene Überakkumulationskrise in den Zentrumsökonomien setzte sich in der europäischen Peripherie in eine Zahlungsbilanzkrise um. Die Krisendynamiken in den beiden europäischen Peripherien mit primärer Prägung durch die Finanziarisierung - Ost- und Südeuropa - folgt zwar ähnlichen Mustern, aber zeitlich versetzt.

Ähnliches lässt sich für die vorgeblichen Anti-Krisen-Politiken feststellen. Während in den EU-Kernländern die dominanten Kräfte zunächst eine massive Stützung des Finanzsektors mit einigen begrenzt dimensionierten anti-zyklischen Maßnahmen kombinierten, verabschiedeten die Regierungen in den finanziarisierten Ökonomien Osteuropas - teils im Rahmen von Programmen mit dem IWF und der EU (Ungarn, Lettland, Rumänien) - bereits im Herbst 2008 strikte Austeritätsprogramme. Diese waren auf die Bewahrung des Wechselkurses gerichtet. Dies lag im Interesse der westeuropäischen Banken, die keine Abwertung ihrer Aktiva in der Region wünschten und die negative Folgen einer Abwertung im Bereich der Fremdwährungskredite fürchteten, aber auch der in Devisen verschuldeten Mittelklasse. Dieser Aspekt der Anti-Krisen-Politik wurde kaum öffentlich in Frage gestellt, war also fast hegemonial (vgl. Becker 2010). Statt auf eine offene Abwertung wurde auf eine euphemistisch als „interne Abwertung“ bezeichnete Politik der Budget-, Pensions- und Lohnkürzungen 
gesetzt. Diese sollte über eine brutale Reduktion der Binnennachfrage die Handelsund Leistungsbilanzdefizite und damit den permanenten externen Finanzierungsbedarf reduzieren. Dies wurde auch erreicht. Gleichzeitig verschärfte sich über die Rezession und steigende Arbeitslosigkeit die Schuldenproblematik.

Das Grundmuster dieser Politik wurde ab dem Frühjahr 2010 auf die südeuropäischen Länder der Eurozone übertragen. Die Rationalität und die Interessenkonstellation in Südeuropa sind ähnlich. Auch hier erfolgt die - allerdings schwächere - Bindung der Mittelklasse über das Geldverhältnis. Große Sektoren der Bevölkerung befürworten die fortgesetzte Mitgliedschaft in der Eurozone aufgrund der denkbaren Auswirkungen auf ihre Depositen und Kredite (deren jeweiliger Umrechnungskurs wäre ein hart umkämpftes Politikum) sowie die erwartbaren monetären Turbulenzen bei einem Austritt bzw. Ausschluss aus der Eurozone. Politisch erweist sich die Eurozonenmitgliedschaft als eine harte „monetäre Restriktion“. Allerdings ist dies keine absolute Restriktion. Inzwischen ist ein Teil des ökonomisch-politischen Establishments in den exportorientierten EU-Kernländern, vor allem in Deutschland, für ein Herausdrängen Griechenlands aus der Eurozone (vgl. Becker/Lesay 2012). Die IWF/EU-Programme haben den ausländischen Banken einen substanziellen Abbau ihrer Griechenland-Engagements ermöglicht. Zumindest implizit wird eingestanden, dass die brutale Sparpolitik einige ihrer Kernziele nicht erreicht. So geht das griechische Leistungsbilanzdefizit - trotz der enormen Rezession - nur ganz langsam zurück (Becker 2012a: 108). Es gibt also weiterhin einen permanenten externen Finanzierungsbedarf. Die Erwartung der BefürworterInnen eines Herausdrängens Griechenlands aus der Eurozone scheint nun zu sein, dass eine Wiedereinführung der nationalen Währung und Abwertung das Leistungsbilanzdefizit tatsächlich längerfristig und damit den externen Finanzierungsbedarf senkt - wenn auch zu massiven kurzfristigen Kosten. Die zentrale Bedeutung der Geldverhältnisse - speziell der Wechselkurse und der Mitgliedschaft in der Eurozone- für die Konzipierung und politische Durchsetzung der Austeritätspolitik wird meines Erachtens von Alex Demirović und Thomas Sablowski unterschätzt.

\section{Ungleiche gesellschaftliche Mobilisierung}

Sie unterschätzen auch die Folgen ungleicher Entwicklung und ungleichzeitiger Krisenphasen für die Mobilisierung gegen die Austeritätspolitiken. In einigen exportorientierten Zentrumsökonomien mit geringer innerer Finanziarisierung (z.B. begrenzter Verschuldung der privaten Haushalte) ist die erste Krisenphase relativ kurz gewesen und durch Maßnahmen wie Kurzarbeit sozial abgemildert worden. In diesen Ländern hat es eine vorübergehende Erholung gegeben. Diese schwächt sich allerdings aufgrund der Krise und scharfen Austeritätspolitik in der europäischen Peripherie bereits deutlich ab. Die Verankerung der Austeritätspolitik und die tiefen Eingriffe in die nationalstaatliche Budget- und Lohnpolitik durch die Veränderungen der ökonomischen Governance in der EU (vgl. Klatzer/Schlager 2011, Bieling 2011) werden trotz der damit verbundenen weiteren Entdemokratisierung der Wirtschaftspolitik in der Öffentlichkeit wenig wahrgenommen und haben bislang keine nennenswerten gesellschaftlichen Mobilisierungen ausgelöst, obgleich es konterkarierende Initiativen auf politischer und juristischer Ebene gibt. Die Etablierung von Formen regelgebundener Wirtschaftspolitik, 
entsprechend der neoliberalen Konzeption Hayeks, ist im Gefolge der Krise wesentlich weiter vorangetrieben worden. Damit sind die Spielräume selbst für eine Wirtschaftspolitik keynesianischen Zuschnitts, von den deutlich weitergehenden Vorschlägen von Demirović und Sablowski ganz zu schweigen, bereits jetzt deutlich weiter eingeschränkt worden. Meines Erachtens unterschätzen die beiden Autoren, wie tief die liberal-autoritäre Transformation der EU-Staatlichkeit reicht. Damit verbleiben den aufEU-Ebene ohnehin organisatorisch und politisch schwachen Gewerkschaften, Linksparteien und progressiven Bewegungen noch weniger institutionelle Anknüpfungspunkte als vor der aktuellen Krise. Die „strategische Selektivität" (Jessop 2002: 40) der europäischen Staatlichkeit ist speziell auf der und über die EU-Ebene weiter zugunsten großer Kapitalgruppen, vor allem im Finanzsektor, verschoben worden. Eine Veränderung dieser „strategischen Selektivität" auf EU-Ebene würde eine sehr starke zeitlich synchrone und auf ein alternatives EU-Projekt setzende Mobilisierung in der EU als eine notwendige, aber nicht hinreichende Bedingung haben. Die sozialen Mobilisierungen gegen die Anti-Krisenpolitik waren jedoch bislang asynchron und im wesentlichen auf die jeweilige nationalstaatliche Ebene begrenzt.

In Osteuropa hat die Austeritätspolitik begrenzte gesellschaftliche Proteste ausgelöst, die wohl am stärksten in Rumänien ausfielen. Es hat Demonstrationen, mehrfach auch Streiks (vor allem im öffentlichen Dienst) gegeben. Im Zentrum der Proteste standen meist die sozialen Folgen der extremen Sparpolitik (vgl. Becker 2010: 535, Dale/Hardy 2011:261). Das Ziel der Wechselkursstabilisierung wurde hingegen kaum öffentlich in Frage gestellt. Die vielleicht am weitesten gehenden politischen Folgen hatten die Delegitimierung der bisherigen
Regierungsparteien und die soziale Unzufriedenheit in Ungarn. Sie brachten dort eine Verschiebung zu Gunsten von Fidesz, die eine Mischung aus Nationalkonservatismus und Neoliberalismus vertritt. Fidesz hat sich als klare Sachwalterin der oberen Mittelklasse profiliert. Hierbei hat sie auch die Problematik der Devisenschulden aufgegriffen und ist - im Gegensatz zu ihrer sozialliberalen Vorgängerregierung - einen begrenzten Konflikt mit den westeuropäischen Banken eingegangen. Den DevisenschuldnerInnen räumte Fidesz die Möglichkeit ein, ihre Fremdwährungsschulden zu günstigeren Wechselkursen, die das Vorkrisenniveau reflektierten, zurückzuzahlen. Die Banken reagierten scharf, da sie einen Präzedenzfall befürchten. Fidesz tangierte hingegen in keiner Weise die Interessen des Auslandskapitals in der Exportindustrie. Trotzdem zeigt der ungarische Fall einen partiellen Riss in der bisherigen Allianz von Auslandskapital und oberer Mittelklasse auf, den im ungarischen Fall die politische Rechte aufgriff (Becker 2012b).

Die Mobilisierung in Südeuropa erfolgte, entsprechend der unterschiedlichen Krisendynamik, zeitlich in der Tendenz später. Ähnlich wie in Osteuropa hatten die Mobilisierungen nationalen Charakter. In allen Ländern kam es zu Streikbewegungen, vor allem im öffentlichen Dienst. Die Streiks waren in Griechenland besonders stark. Sowohl in Spanien als auch in Griechenland spielen gut ausgebildete junge Leute - angesichts der astronomisch hohen Jugendarbeitslosigkeit und fehlender Zukunftsperspektiven - eine wichtige Rolle. Die arbeitslosen bzw. sehr prekär beschäftigten Jugendlichen hatten in Griechenland bereits in den Vorjahren, speziell im Dezember 2008, starke Sozialproteste getragen (vgl. Benlisoy 2012: 9 ff., 58 ff., Fernandez Steinke 2011). In Griechenland, das ein zahlenmäßig besonders 
starkes Kleingewerbe aufweist, aktivierten sich auch Teile der kleinen Selbständigen, die von Deregulierungsmaßnahmen ihrer Gewerbe seitens der EU tangiert sind.

Bislang ist auf politischer Ebene allein in Griechenland die Machtposition der bisherigen Regierungsparteien so stark erodiert, dass deren Regierungsfähigkeit in Frage steht. Syriza hat sich als Linkspartei mit einer eindeutigen Positionierung gegen die Sparpolitik zu einem starken Widerpart zum bisherigen politischen Establishment entwickelt. Bei den Wahlen am 17. Juni wurde sie mit einem Stimmenanteil von $26,9 \%$ knapp hinter der liberal-konservativen Nea Dimokratia die zweitstärkste parlamentarische Kraft. Die nach diesen Wahlen gebildete Dreiparteienkoalition, der Syriza als zentrale politische Kraft der Opposition gegenübersteht, ist jedoch als fragil einzuschätzen.

\section{Die Eurozone als Entwicklungshindernis}

Doch mit dem Bedeutungsgewinn bis hin zum potenziellen Kandidaten als Regierungspartei stellt sich für Syriza jene Frage, bei der Alex Demirović und Thomas Sablowski (2012: 102) für Abstinenz der Linken plädieren: die Mitgliedschaft in der Eurozone. Einerseits sieht sich die griechische Linke seitens wichtiger Sektoren der EU-Kernstaaten, vor allem Deutschlands, mit der Drohung des Ausschlusses aus der Eurozone konfrontiert, sofern Griechenland sich nicht an die Vorgaben zur Austeritätspolitik halte. Das griechische politische Establishment setzte diese Drohung in einem Angstwahlkampf - nach dem Motto: Wir oder das Währungschaos - ein. Der Syriza-Vorsitzende warb zwar in EUKernländern für eine flexiblere Position der $\mathrm{EU}$ in der Politik gegenüber Griechenland. Aber ein ausreichend starkes Gegengewicht besteht aufgrund der ungleichen Mobilisierung im EU-Maßstab nicht.

Die Frage der Mitgliedschaft in der Eurozone ist allerdings auch schon in den Monaten zuvor innerhalb der griechischen Linken und auch innerhalb der SyrizaKoalition kontrovers diskutiert worden. Teile der griechischen Linken erwarten von einem Austritt aus der Eurozone eine Erweiterung von Handlungsspielräumen. Hierbei ist für die BefürworterInnen eines solchen Schritts zuweilen Argentinien eine positive Referenz (vgl. Kouvelakis 2011). Die argentinische Regierung ging in Folge einer tiefen Krise Anfang 2002 vom Currency Board als einem sehr rigiden währungs- und geldpolitischen Arrangement $a b$ und ließ eine starke Abwertung des Peso zu. Die Abwertung stimulierte einerseits die Exporte und ermöglichte andererseits eine Erholung der binnenmarktorientierten Industrieproduktion. Später handelte die argentinische Regierung eine substanzielle Reduktion der Auslandsschulden aus. Diese Politik wurde auch von einem bedeutsamen Sektor des ökonomischen und politischen Establishments getragen. Die argentinische Erfahrung lässt sich allerdings nicht ohne Weiteres auf Griechenland übertragen. Das griechische Establishment hält eindeutig am Euro fest. Die griechische Wirtschaftsstruktur ist weit ungünstiger als die argentinische. Es gibt keinen starken Exportsektor, wie die argentinische Agro-Industrie, und auch die binnenorientierten Industriebranchen sind schwach. Von daher wäre ein Ausscheiden aus der Eurozone weit schwächer innergesellschaftlich abgestützt als die Währungsabwertung in Argentinien. Auch die ökonomischen Impulse wären aus meiner Sicht deutlich schwächer als im Fall Argentiniens (vgl. Becker/Lesay 2012). Sapir (2012: $161 \mathrm{f})$ betont unter Verweis auf die Ex- und Importelastizitäten, dass für praktisch alle 
südeuropäischen Länder (einschließlich Griechenlands) eine Währungsabwertung prinzipiell von Vorteil wäre. Gleichzeitig ist für mich nicht erkennbar, wie es bei den bestehenden Währungsstrukturen in den südeuropäischen Euroländern zu einer Wiederherstellung vor allem binnenorientierter, produktiver Strukturen kommen kann (ähnlich Kaltenbrunner 2012). Eine Stärkung der produktiven Strukturen wäre die Voraussetzung für die Wiedergewinnung wirtschaftspolitischer Handlungsspielräume einer linken Regierung. Dies wäre allerdings kein rascher Prozess und würde begleitend zur Abwertung die Entwicklung einer staatlich induzierten Industriepolitik zur Voraussetzung haben.

Eine relevante industriepolitische Diskussion scheint es in Syriza bislang nicht zu geben. Das Wahlprogramm war auf Fragen der Verschuldung, Vergesellschaftung des Bankensektors, Bewahrung der Sozialstaatlichkeit zentriert (vgl. Semercioğlu 2012, Tsipras 2012), die massiven Ursachen der Krise auch in den Defiziten des produktiven Sektors wurden nicht thematisiert. Ähnliches lässt sich für die spanischen Indignados feststellen (¡Democracia Real YA! 2011). Hierin spiegelt sich möglicherweise das relativ geringe Gewicht der Industriearbeiterschaft in den drei südeuropäischen Ländern, speziell in Griechenland, wider. Die Frage nach den Veränderungen der produktiven Strukturen wird auch von Alex Demirović und Thomas Sablowski - im Gegensatz zu den Eigentumsfragen - nicht thematisiert. Sie äußern die Vermutung, dass sich durch einen Austritt aus der Eurozone an den „extrem ungleichen Produktionsstrukturen" wenig ändern würde (Demirović/Sablowski 2012: 99 f.). Die Frage ist allerdings, ob sich ohne Änderung der Währungsverhältnisse an den Produktionsstrukturen Wesentliches ändern kann. Ich bin skeptisch, dass eine solche Änderung ohne Protektionsmechanismen wie die Währungsabwertung möglich ist. Ich halte auch weniger die von Alex Demirović und Thomas Sablowski angeschnittene Frage einer Exportsteigerung durch eine Abwertung als die Frage der Ersetzung der Importe durch einheimische Produktion für vorrangig. Aus meiner Sicht müsste es um eine Stärkung der binnenorientierten Sektoren gehen. Angesichts der depressiven Ausgangslage würde ich, im Gegensatz zu den beiden Autoren, den inflationären Schub für begrenzt halten. Für den griechischen Bankensektor wäre die Abwertung allerdings sehr kompliziert und eine Regierung wäre zu massiven Eingriffen in den Kapital- und Zahlungsverkehr gezwungen. Eine weitere Schuldenreduktion wäre unvermeidlich.

Der politische Konflikt spitzt sich letztlich an der Frage der Geldnorm zu. Sollte die Linke in einem der Peripherieländer an die Regierung kommen, würde sich für sie nicht allein die Frage nach der Mitgliedschaft in der Eurozone, sondern auch der Veränderung der Produktionsstruktur stellen. Außenwirtschaftliche Spielräume und Verhandlungsmacht können nur über eine Minderung der Importabhängigkeit gewonnen werden.

Solange linke Kräfte in der Defensive sind, ist der von Alex Demirović und Thomas Sablowski vorgeschlagene gemeinsame Fokus auf der Abwehr der Austeritätspolitik plausibel. Allerdings stellen sich schon in dieser Phase die Fragen in der Peripherie etwas anders als in den Zentrumsökonomien, da die vorherrschende monetäre Norm in ersterer Deindustrialisierung und hohe strukturelle Arbeitslosigkeit begünstigt. Derzeit sind die gesellschaftlichen und politischen Widerstände in einzelnen Peripherieländern am stärksten. Dort stellt sich angesichts der ungleichen Entwicklung und ungleichen Mobilisierung bei einer Zuspitzung der politischen Konflikte die Frage nach der Gewinnung nationalstaatlicher Spielräume 
für eine alternative Politik. In Griechenland ist dieser Punkt bereits mit den Wahlen vom 6. Mai erreicht worden. Dieser Frage gehen Alex Demirović und Thomas Sablowski aus dem Weg. Meines Erachtens hätte die Linke in den EU-Zentrumsstaaten eine Entscheidung von Linkskräften in der EU-Peripherie, aus der Eurozone auszutreten, als legitim anzuerkennen und ihnen für Alternativansätze soviel Unterstützung zu geben, wie es in ihren Kräften steht.

Mehrheitlich neigt allerdings Syriza momentan - wohl nicht zuletzt aufgrund der erheblichen kurzfristigen Folgen eines Austritts aus der Eurozone und aufgrund der starken Befürwortung einer Beibehaltung des Euro in breiten Bevölkerungsgruppen einem Verbleib in der Eurozone zu. Dies ist eine legitime Positionierung. Sie scheint mir allerdings nicht realistisch. Zum einen stehen dem die politischen Kräfteverhältnisse entgegen. Starke Kräfte in den Kernländern der Eurozone setzen ohnehin auf einen Ausschluss Griechenlands - ohne auch nur in Ansätzen Vorstellungen über eine Entwicklungsstrategie Griechenlands nach einer Abwertung erkennen zu lassen. Syriza könnte dann versuchen, die Zuspitzung des Konflikts zum Katalysator für ein alternatives EU-Projekt zu machen. Allerdings - und das ist das zweite Problem - ist die Frage offen, inwieweit bei einem Verbleib der peripheren Eurozonenländer die Umkehrung der ungleichen Entwicklung bzw. „Entwicklung der Unterentwicklung" (Frank 2008) überhaupt möglich ist. Meines Erachtens sind die ungleichen Entwicklungsmuster bereits soweit fortgeschritten, dass selbst eine starke Förderung der binnenorientierten industriellen Entwicklung und eine stärker auf die Protektionsnotwendigkeiten der Peripherie zugeschnitteneEU-Außenwirtschaftspolitik die ungleichen Entwicklungsmuster nur mehr abmildern, nicht aber umkehren könnten. Eine solche Veränderung wird selbst unter linken EU-ÖkonomInnen der EuroMemo Gruppe erst seit sehr kurzer Zeit diskutiert (vgl. EuroMemo Gruppe 2012: 56) und spielt bei den ansonsten sehr breit angelegten politischen Überlegungen von Alex Demirović und Thomas Sablowski keine Rolle. Meines Erachtens wäre für die peripheren EU-Länder, möglicherweise aber sogar auch für Frankreich (vgl. Sapir 2012: 162,176 ff.), über eine veränderte Entwicklungspolitik und Außenwirtschaftspolitik hinaus zumindest ein monetärer Schutzmechanismus - in Form der Möglichkeit der Währungsabwertung - erforderlich.

Einer selbst bescheidenen Neuausrichtung der EU-Wirtschaftspolitik - im Sinne einer wenigstens expansiven Lohn- und Fiskalpolitik in den Zentrumsländern sowie einer industriellen Regionalpolitik in der Peripherie - stehen zudem durch die bereits weit vorangeschrittene Etablierung regelgebundener Wirtschaftspolitik im Rahmen der liberal-autoritären Transformation der EU gewaltige institutionelle Hindernisse entgegen. Angesichts der „strategischen Selektivität" der EU und der aufEU-Ebene besonders ungünstigen Kräfteverhältnisse für progressive Kräfte der Arbeiterschaft und Mittelschichten ist es fraglich, inwieweit eine in einem progressiven Sinne ,andere EU“ überhaupt möglich ist.

\section{Literatur}

Becker, Joachim (2007): Dollarisation in Latin America an Euroisation in Eastern Europe: Parallels and Differences. In: Becker, Joachim/ Weissenbacher, Rudy (Hg.): Dollarization, Euroization and Financial Instability. Central and Eastern Europe between Stagnation and Financial Crisis? Marburg: 223-278

- (2010): Krisenmuster und Anti-KrisenPolitiken in Osteuropa. In: Wirtschaft und Gesellschaft, 36. Jg., Nr. 4: 517-540

- (2012a): Die Linke und die Euro-Zone. In: Kurswechsel, Nr. 1: 108-113 
- (2012b): Neo-liberalism's new cloth: national conservatism in Hungary and Turkey. In: Sendika.org, 14.4., www.sendika.org/english/ yazi-no=44311 (abgerufen 9.5.2012)

Becker, Joachim/Jäger, Johannes (2009): Die EU und die große Krise. In: PROKLA, 39.Jg., Nr. 4: $541-558$

- (2010): Development Trajectories and the Crisis in Europe. In:Debatte: Journal of Contemporary Central and Eastern Europe, 18. Jg., Nr. 1: 5-27

Bellofiore, Riccardo/Garibaldo, Francesco/ Halevi, Joseph (2010): The global crisis and the crisis of European neo-mercantilism. In: Panitch, Leo/Albo, Greg/Chibber, Vivek (Hg.): Socialist Register 2011: the crisis this time. London u.a.O.: 120-146

Benlisoy, Foti (2012): 21. Yüzyılın İlk Devrimci Dalagasi. Fransa ve Yunanistan'dan Arap Devrimi, "The Occupy" Hareketleri ve Kürt Isyanina. Istanbul

Bieling, Hans-Jürgen (2011): EU-Verfassungspolitik und Wirtschaftsregierung. Konstitutionalismus gegen Volkssouveranität und Demokratie. In: Widerspruch, 31. Jg, Nr. 2: 61-70

Dale, Gareth/Hardy, Jane (2011): Conclusions: the "Crash" in Central and Eastern Europe. In: Dale, Gareth (Hg.): First the Transition, then the Crash. Eastern Europe in the 2000s. London: 251-263

Demirović, Alex/Sablowski, Thomas (2012): Finanzgetriebene Akkumulation und die Krise in Europa. In: PROKLA, 42. Jg., Nr. 1: 77-106

¡Democracia Real YA! (2011): Propuestas. In: Taibo, Carlos et al.: La rebelión de los indignados. Movimiento 15 M: Democracia real, iya! Madrid: 87-92

EuroMemo Gruppe (2012): EuroMemo 2012. Europäische Integration am Scheideweg: Mehr Demokratie für Stabilität, Solidarität und soziale Gerechtigkeit. Supplement der Zeitschrift Sozialismus: 3/2012

Fernandez Steinko, Armando (2011): Le mouvement 15-M en Espagne. In: Savoir/agir, Nr. 18: 55-66

Fouskas, Vassilis K. (2012): Insight Greece: The Origins of the Present Crisis. In: Insight Turkey, 14. Jg., Nr. 2: 27-36

Frangakis, Marica (2009): Europe's financial system under pressure. In: Grahl, John (Hg.): Global Finance and Social Europe. Cheltenham: 53-90

Frank, André Gunder (2008): Die Entwicklung der Unterentwicklung (1966). In: Fischer, Karin/
Hödl, Gerald/Sievers, Wiebke (Hg.): Klassiker der Entwicklungstheorie. Von Modernisierung bis Post-Development. Wien: 147-167

Hrvatska narodna banka (2011): Financijska stabilnost. Nr. 7

Jessop, Bob (2002): The Future of the Capitalist State. Cambridge

Kaltenbrunner, Annina (2012): Strukturelle Ungleichgewichte und Austerität. Gibt es eine Zukunft für periphere Länder in der Eurozone? In: Kurswechsel, Nr. 2: 114-121

Klatzer, Elisabeth/Schlager, Christa (2011): Europäische Wirtschaftsregierung - eine stille neoliberale Revolution. In: Kurswechsel, $\mathrm{Nr}$. 1: 61-81

Kouvelakis, Stathis (2011): The Greek Cauldron. In: New Left Review, Nr. 72: 17-33

López, Isidro/Rodríguez, Emmanuel (2010): Fin de ciclo. Financiarización, territorio y sociedad de propietarios en la onda larga del capitalismo bispano (1959-2010). Madrid

Rodrigues, João/Reis, José (2012): The Asymmetries of European Integration and the Crisis in Portugal. In: Competition and Change, 16. Jg., Nr. 3 (i.E.)

Santos, Vitor/Jacinto, Ana (2006): A industria portuguesa: Desindustrialização - progresso ou declinio? In: Romão, António (Hg.): $A$ economia portuguesa 20 anos após a adesão. Coimbra: 184-213

Sapir, Jacques (2012): Faut-il sortir de l'euro? Paris

Semercioğlu, Can (2012): Bankalar kamulaştırılacak, NATO'dan çıkılacak. In: Bir Gün, 26.5.:10

Stathakis, George (2010): The fiscal crisis of the Greek economy. In: Kurswechsel, Nr. 3: 109-114

Tsarouhas, Dimitris (2012): The Political Origins of the Greek Crisis: Domestic Failures and the EU Factor. In: Insight Turkey, 14. Jg. Nr. 2: 83-98

Tsipras, Alexi (2012): Regierungsprogramm der Radikalen Linken SYRIZA in Griechenland. In: www.griechenland-blog.gr/2012/Regierungsprogramm-der-radikalen-linken-syrizain-griechenland/(abgerufen 2.7.2012)

Weissenbacher, Rudy (2007): Historical Considerations on Uneven Development in East Central Europe. In: Becker, Joachim/Weissenbacher, Rudy (Hg.): Dollarization, Euroization and Financial Instability. Central and Eastern European Countries between Stagnation and Financial Crisis? Marburg: 35-83 